\title{
Virus-free and oncogene-free induced pluripotent stem cell reprogramming in cord blood and peripheral blood in patients with lung disease
}

\author{
Anant Kamath ${ }^{1,2}$, Sara Ternes ${ }^{2}$, Stephen McGowan ${ }^{3}$ \& Alan B Moy*,1,2,4 \\ ${ }^{1}$ Cellular Engineering Technologies (CET), Inc., Coralville, IA, 52241, USA \\ ${ }^{2}$ The John Paul II Medical Research Institute (JP2MRI), lowa City, IA, 52241, USA \\ ${ }^{3}$ Division of Pulmonary, Critical Care \& Occupational Medicine, University of lowa, lowa City, IA, 52242, USA \\ ${ }^{4}$ Department of Biomedical Engineering, University of lowa, lowa City, IA, 52214, USA \\ *Author for correspondence: moya@celleng-tech.com
}

Aim: A virus- and oncogene-free induced pluripotent stem cell (iPSC) reprogramming method was developed with cord blood-derived mononuclear cells (CBDMNC) and peripheral blood mononuclear cells (PBMNC) from patients with genetic lung diseases. Method: iPSC reprogramming used small molecules, hematopoietic stem cell (HSC) expansion media and episomal vectors that lacked Myc and Lin28. Results: All iPSC colonies were fully reprogrammed based on SSEA4 expression. A total of 300,000 CBDMNC was the optimal cell number for cell reprogramming, which was associated with a 13-fold increase in CD34+ cells upon exposure to HSC media. Cell reprogramming was not observed in the absence of HSC expansion media. The method also reprogrammed PBMNC in patients with cystic fibrosis or $\alpha-1$ antitrypsin deficiency. Oncogene-free iPSC cell lines differentiated into all three germ cell lineages. Conclusion: This iPSC reprogramming approach satisfies an important regulatory requirement for iPSC-based cell therapies with lower clinical risk from CBDMNC and PBMNC.

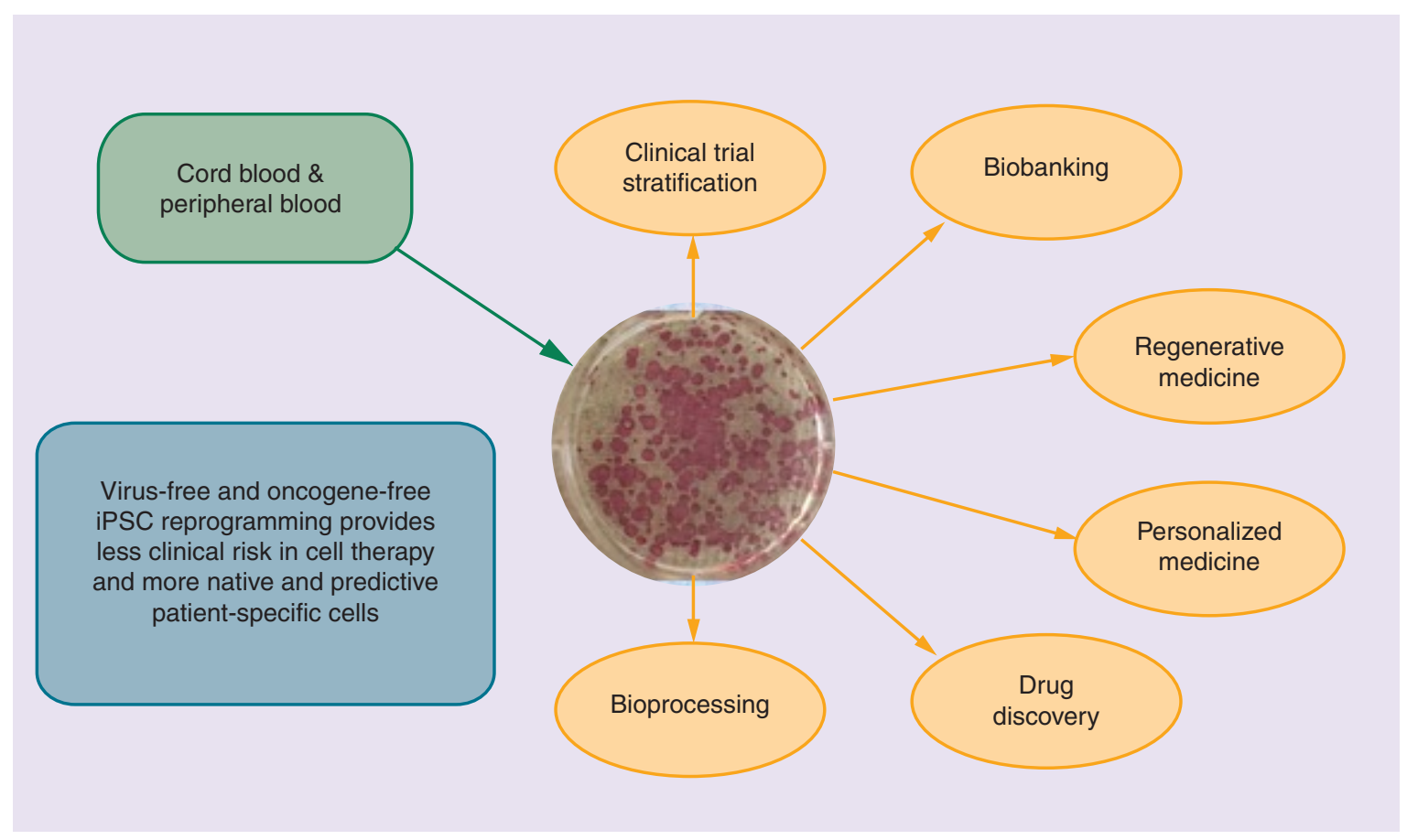

Future Medicine 
First draft submitted: 5 April 2018; Accepted for publication: 2 November 2018; Published online: 29 November 2018

Keywords: $\alpha-1$ antitrypsin deficiency $\bullet$ CD34+ • cystic fibrosis • episomal • hematopoietic stem cell • iPS cell • mononuclear cells • reprogramming

It has been a common practice to privately bank cord blood as a strategy to provide a source of autologous hematopoietic stem cells (HSC) if a child acquires a blood or metabolic disorder later in childhood or if a sibling requires a HLA-matched donor. However, there have been criticisms with the common practice of privately banking cord blood as a future healthcare insurance [1]. First, the chances are extremely rare that privately banked cord blood will be used for later autologous cell therapy or allogeneic cell therapy for a sibling during childhood [1]. Second, bone marrow transplant centers will typically not perform autologous cord blood transplants for childhood leukemia because of the likelihood that such banked cord blood would contain preleukemic mutations [2]. Third, there is an insufficient number of HSC in each unit of cord blood to treat that same individual later during adulthood [3]. Fourth, HSC do not have the capacity to differentiate into all germ cell lineages [4]. Thus, adults lack good options to store master stem cell stocks for autologous cell therapy for future use if that individual later develops a chronic disease. Yet, conversion of mononuclear cells (MNC) from cord blood and peripheral blood into induced pluripotent stem cells (iPSC) could solve these problems for several reasons. First, iPSC has a significant scalability. Second, iPSC would extend the lifetime utility of cord blood to later produce an autologous cell therapy. Third, iPSC would provide an autologous source of pluripotent stem cells to treat several chronic conditions other than blood disorders such as cancer, macular degeneration, cardiopulmonary disease, central nervous system disorders, metabolic diseases, and chronic liver and kidney disorders. Fourth, cord blood-derived CD34+ cells would express the least number of mutations. Fifth, autologous iPSC would avoid the immunogenicity from HLA mismatch between donor and recipient inherent in allogeneic bone marrow transplantation. Last, autologous iPSC would avoid the risk of opportunistic infection caused by the immunosuppressive agents required in allogeneic HSC transplantation [5].

Takahashi et al. were the first group to demonstrate the dedifferentiation of somatic fibroblasts into pluripotent stem cells through retroviral gene delivery of Oct3/4, Sox2, Klf4 and c-Myc [6,7]. Yu et al. also reported creating cultured iPSC from fetal and neonatal fibroblasts by retroviral delivery of Oct3/4, Sox2, Nanog and Lin28 [8]. Nakagawa and Yamanaka et al. further observed that deletion of c-Myc from the reprogramming scheme still created pluripotent colonies but eliminated teratoma formation [9]. In a follow-up report, Nakagawa and Yamanaka demonstrated that replacement of c-Myc with 1-Myc eliminated the neoplastic effects associated with c-Myc [10] when reprogramming was conducted with retroviral systems. Yet, 1-Myc has been associated with several clinical malignancies [11-13]. Zhao previously reported teratoma formation from iPSC created with episomal vectors that contained 1-Myc/Lin28 [14]. Taken together, these data indicate that the oncogenes, c-Myc and Lin28, are the chief determinants of the neoplastic risk associated with current iPSC reprogramming methods.

A previous report documented an episomal reprogramming method for creating a nonintegrating, virus-free and Lin28 and Myc-free iPSC cells in adherent fibroblasts by using a mixture of reprogramming small molecules and episomal vectors [15]. The use of these reprogramming molecules was robust enough to produce fully reprogrammed iPSC colonies even in the absence of c-Myc or a combination of l-Myc/Lin28. The report demonstrated that the iPSC reprogramming efficiency was equal to or exceeded that of prior reports that used episomal expression systems dependent on the expression of these oncogenes [16,17]. Also, 100\% of oncogene-free colonies were fully reprogrammed regardless in the presence or absence of oncogenes.

The goal of this study was to adapt a Myc/Lin28-free iPSC reprogramming method for cord blood and peripheral blood, which could potentially facilitate new opportunities in autologous and allogeneic cell therapy. The prior Myc and Lin28-free iPSC reprogramming approach was further optimized to convert MNC from cord blood and peripheral blood from patients with genetic lung diseases to serve as cell models of disease without the potential viral and oncogenic perturbations that could skew the predictability of drug discovery applications. This report addressed the following questions: is the combinatorial approach of oncogene-free iPSC reprograming sufficient to produce iPSC colonies?; if not, what modifications are required to produce iPSC colonies?; what is the reprogramming efficiency of Myc/Lin28-free iPSC in cord blood-derived mononuclear cells (CBDMNC)?; what is the minimum number of CBDMNC required to achieve a sufficient number of iPSC colonies?; what fraction of the colonies 
are fully reprogrammed?; do Myc/Lin28-free iPSC differentiate into all three germ cell lineages characteristic of pluripotent stem cells?

Last, this study evaluated whether Myc/Lin28-free iPSC reprogramming is robust enough to develop patient and disease-specific iPSC from peripheral blood from patients with genetic diseases, which could produce a more native iPSC that could provide more predictive drug efficacy and safety or used as an autologous cell therapy. For this study, genetic lung diseases such as cystic fibrosis (CF) and $\alpha-1$ antitrypsin deficiency (A1ATD) were chosen. This study reports the first virus-free and oncogene-free iPSC developed from a child with CF expressing the homozygous $\delta-508$ mutation and an adult patient with A1ATD expressing a ZZ phenotype.

\section{Methods}

\section{Cultured iPSC from human foreskin fibroblasts}

An established cell line previously developed from iPSC reprogramming of cultured human foreskin fibroblasts (HFF-iPSC) [15] was used to differentiate in all three germ cell lineages.

\section{iPSC differentiation}

iPSC were reprogrammed into ectoderm (neuronal phenotypes), definitive endoderm (DE) and mesoderm.

For neuronal differentiation, iPSC were disaggregated into clumps using versene and plated on tissue culture dishes coated with Laminin and Ornithine. Attached iPSC clusters were treated with neuronal differentiation media (Cellular Engineering Technologies [CET], IA, USA). Cells were fed every 48 h. Rosette formation was seen after $72 \mathrm{~h}$. Neuronal phenotype was confirmed with in situ immunohistochemistry for Nestin and Pax-6 expression.

For DE differentiation, iPSC were disaggregated using versene to form clumps that contained 200-300 cells. Clumps were plated on tissue culture dishes using definitive endoderm differentiation media (CET, Coralville, IA). Cells were fed every 48 hours. At the end of 5 days, cells were subjected to immunohistochemistry for FOX-A2 and Sox-17, which are markers of DE.

For mesodermal differentiation, iPSC were disaggregated using versene to form clumps of 200-300 cells. Clumps were plated on tissue culture dishes using mesoderm induction media (CET, IA, USA). Cells were fed every 48 h. At the end of 5-days, cells were subjected to immunohistochemistry for brachyury, a known mesodermal marker. The iPSC were differentiated into cardiomyocytes using a cardiac differentiation media (CET). Cardiomyocyte differentiation was confirmed by expression of cardiac troponin.

For lung progenitor differentiation, CD34+ iPSC were treated with versene and plated on fresh Vitronectin-XF at a density of about $1200 \mathrm{cells} / \mathrm{cm}^{2}$. At the end of the first $24 \mathrm{~h}$, a complete media change was done to differentiate iPSC into definitive endoderm differentiation media (CET). Cells were fed every $48 \mathrm{~h}$ for a total of 6 days. At the end of 6 days, DE media was replaced with anterior foregut (AF) media (CET). Cells were maintained in AF media for a period of $48 \mathrm{~h}$ for AF patterning. At the end of $48 \mathrm{~h}$, cells were treated with versene, dislodged and clump passaged on freshly coated geltrex plates at $1200 \mathrm{cells} / \mathrm{cm}^{2}$. Cells were then fed with lung progenitor induction media (CET). Completed media exchanges with the lung progenitor induction media were conducted every 48 h. After a period of 14 days in this media, cells were evaluated for lung differentiation by probing them with a monoclonal antibody specific for cytokeratin 5/8 expression (Santa Cruz Biotechnology, TX, USA).

\section{Purification \& expansion of CD34+ cells from cord blood}

Red blood cells were depleted from cord blood using a starch-based RBC cross linking reagent (Hetasep, Stem Cell Technologies Catalog \# 07806, Vancouver, British Columbia, Canada) according to manufacturer's directions. Nucleated, leukocyte rich fractions, were resuspended in $1 \mathrm{ml}$ of Dulbecco's phosphate-buffered saline (DPBS, ThermoFisher Scientific, MA, USA) for subsequent steps. CD34+ cells were isolated from the leukocyte rich fraction using antibody-based separation. Briefly, CD34+ cells were positively selected using Miltenyi’s CD34+ Microbead Kit (Miltenyi Catalog \# 130-046-703, CA, USA), which contains magnetic beads that are conjugated to monoclonal antibody specific for CD34+ antigen. Positive selection was conducted twice serially to increase purity of isolated CD34+ cells. CD34+ cells were grown in CET's HSC growth media using low attachment dishes. CD34+ cells were pelleted every 3 days by centrifugation at $100 \times \mathrm{g}$ for $5 \mathrm{~min}$ at room temperature. They were then transferred to fresh low-attachment dishes every $72 \mathrm{~h}$. 
Table 1. Summary of normal and disease-specific induced pluripotent stem cell from various tissue sources: table summarizes the tissue sources used to create normal and disease-specific, virus-free and oncogene-free induced pluripotent stem cell.

\begin{tabular}{|c|c|c|c|c|}
\hline iPSC cell line & Tissue source & Sample size & CD34+ purified & HSC expansion \\
\hline Normal & Cord blood & 3 & Yes & Yes \\
\hline Normal & CBDMNC & 1 & No & Yes \\
\hline Normal & HFF & 3 & $\mathrm{~N} / \mathrm{A}$ & No \\
\hline$\delta-508 \mathrm{CF}$ & PBMNC & 1 & No & Yes \\
\hline A1ATD (ZZ) & PBMNC & 1 & No & Yes \\
\hline
\end{tabular}

These derived induced pluripotent stem cells were created from purified normal cord blood-derived CD34+ cells; CBDMNC; HFF; and peripheral blood from a patient with CF and A1ATD. A1ATD: $\alpha-1$ anti-trypsin deficiency; CBDMNC: Cord blood-derived mononuclear cell; CF: Cystic fibrosis; HFF: Human foreskin fibroblast; HSC: Hematopoetic stem cell; PBMNC: Peripheral blood mononuclear cell.

Isolation of MNC from cord blood \& peripheral blood

Cultured neonatal MNC were isolated from discarded cord blood. Cord blood from full-term healthy newborns was obtained under informed consent from parents that underwent routine normal deliveries from a local hospital. Additionally, $5 \mathrm{ml}$ of whole blood were obtained by venipuncture from a 7-year-old male CF patient that carried a homozygous $\delta$-508 mutation. A similar amount of blood was obtained from a 57-year-old female with A1ATD carrying a ZZ-phenotype. Clinical procedures were approved by the John Paul II Medical Research Institute Institutional Review Board (IRB). A list of the number of iPSC derived from cord blood, HFF and patients with genetic lung disease is depicted under Table 1. The data also documents that cord blood-derived CD34+ and peripheral blood is pretreated with HSC expansion media. Isolated cultured cells were deidentified in accordance with Institutional Review Board procedures such that researchers who processed tissue samples were not aware of the donor's identity. Whole blood was collected during venipuncture using standard vacutainer tubes containing EDTA. Whole blood was diluted 1:1 using DPBS. Peripheral blood mononuclear cells (PBMNCs) were isolated using the Ficoll-Paque technique density-based centrifugation. Briefly, $16 \mathrm{ml}$ of Ficoll-Paque solution was pipetted into a Leucosep tube. The tube was spun at $1000 \times \mathrm{g}$ for $30 \mathrm{~s}$ at $20^{\circ} \mathrm{C}$. The Ficoll-Paque was located below the porous barrier. The diluted whole blood was layered above the styrofoam frit in the Leucosep tube and the sample was spun at $1000 \times \mathrm{g}$ for $10 \mathrm{~min}$ at $20^{\circ} \mathrm{C}$. The middle (white) layer, consisting of PBMNCs, was collected using a serological pipette. The collected layer was mixed with an equal volume of DPBS, mixed and centrifuged at $300 \times \mathrm{g}$ for $10 \mathrm{~min}$ at $20^{\circ} \mathrm{C}$. The supernatant was aspirated and cells were resuspended in $300 \mu \mathrm{l}$ of DPBS and counted using a Millipore Scepter counter. The MNC fraction was then used downstream to genetically reprogram into iPSC.

\section{Episomal vector production}

Each vector containing a gene of interest was based on the Invitrogen's pCEP-4 episomal vector previously reported [15] (Figure 1). Briefly, each vector contains an Epstein-Barr virus origin of replication, SV40 poly adenylation sequence, $2 \mathrm{~A}$ cleavage sequence for tandem multicistronic genes, a bacterial origin of replication and an ampicillin/hygromycin antibiotic resistance genes. Each vector either contains a single gene or tandem genes separated by a $2 \mathrm{~A}$ cleavage sequence. In addition to the traditional Yamanaka factors (Oct3/4, Sox2 and Klf4), there are separate vectors containing l-Myc along with Lin28, along with separate episomal vectors that encode for p53 antisense RNA and c-Myc. The system also contains a vector that encodes for red fluorescent protein to monitor efficiency of gene delivery and to detect silencing of exogenous reprogramming factors. Last, for each of the vectors to efficiently remain in the cell cytoplasm for only a short time frame, the plasmid vector also encoded for Epstein-Barr nuclear antigen-1 (EBNA-1). Three separate iPSC groups were created with cultured CBDMNC, which were reprogrammed with c-Myc; a combination of 1-Myc and Lin28; and one free of Myc and Lin28. The specific vectors and reprogramming molecules used under each condition are depicted in a table (Figure 1).

\section{iPSC reprogramming \& colony expansion}

A temporal sequence of the iPSC reprogramming and cell expansion process is depicted in Figure 1. Isolated cord blood or PBMNC were exposed to 7 days of hematopoietic stem cell expansion media (CET). After this step, prior to transfection, a 6-well dish was coated with Vitronectin-XF according to manufacturer's directions (Primorigen, Madison, WI). MNC were examined under a microscope to ensure logarithmic growth phase and $80 \%$ confluency. 


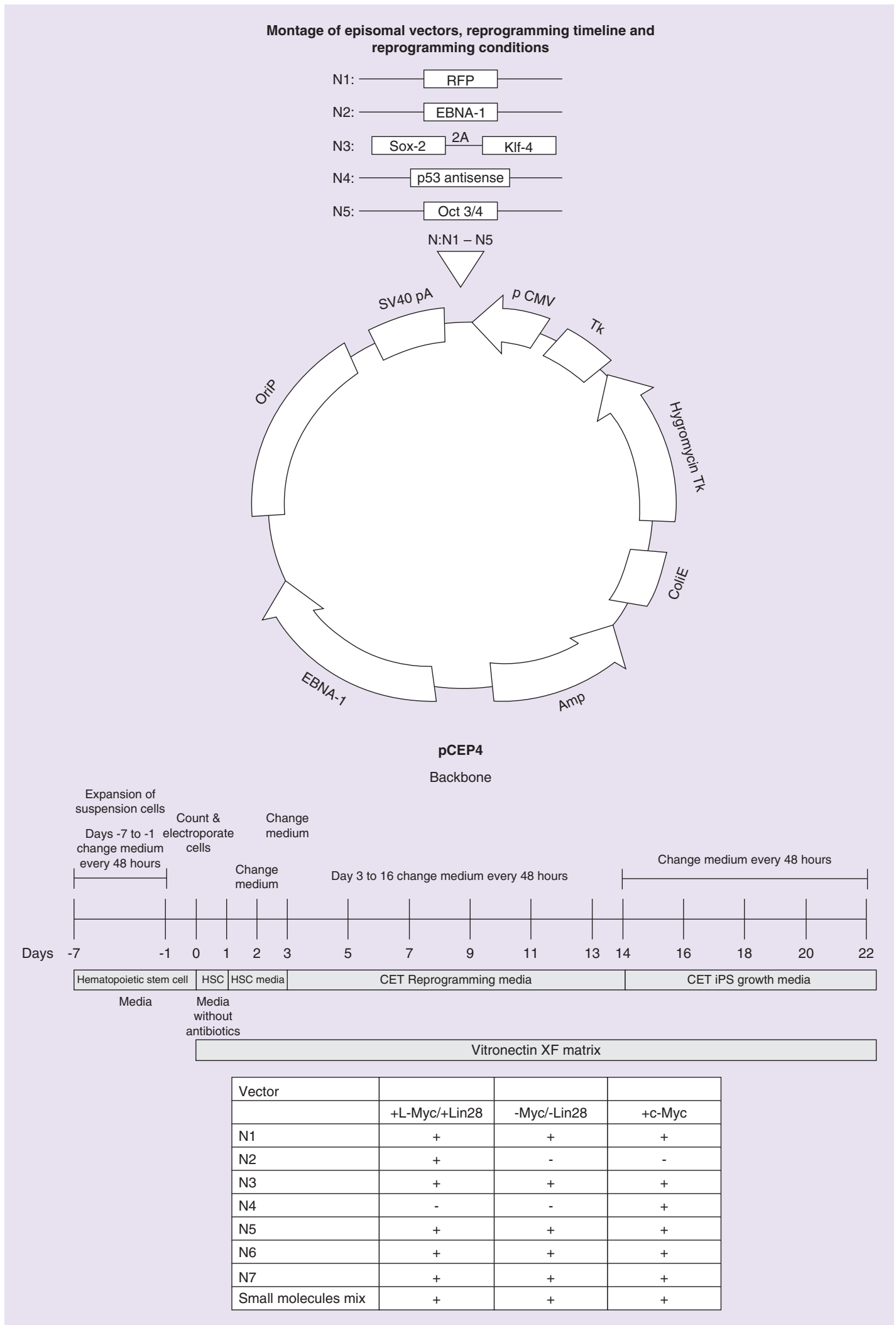

Figure 1. Vector maps for the various episomal constructs. (A) Generalized vector map of the episomal vector. Each vector is based on the pCEP-4 episomal backbone containing an Epstein-Barr virus origin of replication, SV40 poly adenylation sequence, 2A cleavage sequence for tandem genes, a bacterial origin of replication and ampicillin/hygromycin resistance genes. Each vector either contains a single gene or tandem genes separated by a 2A cleavage sequence. See text for details. (B) Figure depicts the time line of HSC pretreatment, iPSC reprogramming and iPSC expansion. (C) A table includes the mixture of episomal vectors and reprogramming molecules that are used for iPSC reprogrammed with c-Myc; a combination of I-Myc and Lin28; and one free of Myc and Lin28. CET: iPSC reprogramming media; HSC: Hematopoietic stem cell; iPSC: Induced pluripotent stem cell. 
Cells were washed with DPBS. MNC were counted and then were adjusted to different densities according to different experimental designs. Cells were spun to pellet at $200 \times \mathrm{g}$ for $5 \mathrm{~min}$. Each cell pellet was resuspended in $100 \mu \mathrm{l}$ of Neon Electroporation Buffer R (ThermoFisher Scientific, MA, USA). $3.5 \mu \mathrm{g}$ of DNA of the author's episomal reprogramming mix was added to each tube and mixed gently. A Neon Electroporation Tip-100 was used to introduce the cells to the DNA. Using Buffer E2 for the chamber buffer, the cells were electroporated at $1650 \mathrm{~V}$ for $10 \mathrm{~ms}$ for three cycles. Immediately after electroporation, the cells were placed in HSC media containing no antibiotics/antifungals on the previously coated 6-well dish for the first $24 \mathrm{~h}$.

After $24 \mathrm{~h}$, the growth media was withdrawn and replaced with iPSC reprogramming media (CET) containing antibiotics/antifungals as previously described [15]. To evaluate successful transfection, cells were examined under a microscope to detect RFP fluorescence within the first $48 \mathrm{~h}$. Cells were fed with fresh iPSC reprogramming media every $48 \mathrm{~h}$ through day 14 of the reprogramming process. From day 15 onwards, a full media replacement was performed every $24 \mathrm{~h}$ with iPSC growth media (CET). Mature iPSC colonies were observed starting around day 17 postelectroporation, which displayed sharp and distinct borders. The identity of the iPSC colonies was confirmed with positive probes for various iPSC markers including SSEA-4, Nanog, Oct4 and TRA160 Live Stain (ThermoFisher Scientific, MA, USA) and alkaline phosphatase (Stemgent, MA, USA).

\section{Flow cytometry}

Flow cytometry was conducted using a Guava EasyCyte HT, MA, USA. Cells were dissociated using trypsin-like enzyme (Tryp-LE) for $10 \mathrm{~min}$ at $37^{\circ} \mathrm{C}$. Dissociated cells were pipetted to remove aggregations and clumps and passed through a 70-micron filter. Single cell suspensions were counted using a Millipore Scepter counter and cell density was adjusted to $1 \times 10^{5}$ cells $/ 100 \mu \mathrm{l} .5 \mu \mathrm{l}$ of appropriate antibody was added to the dissociated cells and mixed using gentle pipetting. Cell-antibody complex was then incubated in the dark for $30 \mathrm{~min}$ on ice. At the end of this incubation period, labeled cells were washed with ice-cold DPBS and resuspended in $200 \mu \mathrm{l} \mathrm{DPBS}$. Cells were then counted using a Guava EasyCyte HT. Viable cells were gated using a $\log / \log$ forward scatter/side scatter plot. Each iPSC marker fluorescence was also compared with its Ig-א isotype control to quantify nonspecific and autofluorescence events. Each iPSC marker was counted and plotted as a graph with the abscissa containing the log fluorescence of a given marker and the ordinate containing the counts of either a negative or positive viable gated cell. This graph was then used to create histograms providing percentages of negative and positive cells. Based on the Ig- $\kappa$ isotype control, $10^{2}$ was used as the cutoff in log fluorescence between a negative and a positive cell.

\section{Statistical analysis}

Data are reported as means $\pm \mathrm{SE}$. Comparisons between more than two groups were made with analysis of variance. Individual group comparisons were done with Tukey's honest significant difference test for post hoc comparison of means. Differences were considered significant at the $\mathrm{p} \leq 0.05$ level.

\section{Results}

Myc- \& Lin28-free iPSC differentiate into all germ cell lineages from HFF-derived iPSC

Reprogrammed iPSC were validated for their pluripotency by differentiating into germ cell lineage cultured cells. Figure 2 represents a montage of HFF-iPSC differentiated into three germ cell lineages. HFF-iPSC were differentiated into cultured mesodermal-derived cells based on expression of brachyury (a biomarker of mesoderm differentiation) and cardiomyocytes based on cardiac troponin. HFF-iPSC also differentiated into cultured DE cells based on expression of Sox 17 and FoxA2. HFF-iPSC differentiated into neuroprogenitor cells based on expression of Pax6 and Nestin. Taken together, these data confirm that Myc/Lin28-free reprogrammed HFF-iPSC exhibit pluripotency based on their ability to differentiate into all three germ cell lineages.

\section{Myc- \& Lin28-free iPSC reprogramming of expanded \& purified CD34+ cells from cord blood}

CD34+ cells were positively selected in cord blood from normal donors by robotic magnetic cell sorting. Purified CD34+ cells were further exposed to HSC expansion media for 1 week and then subjected to Myc/Lin28-free iPSC reprogramming as previously described [15]. Figure 3A depicts a montage of images demonstrating positively selected CD34+ cells expanded after 1 week and subsequently converted into cultured iPSC colonies as depicted by alkaline phosphatase staining and expression of SSEA4, Nanog, Oct4 and TRA160. Taken together, these data demonstrate that the combinatorial approach of small reprogramming molecules and episomal vectors free of Myc/Lin28 were sufficient to reprogram purified cord blood derived CD34+ cells. 
Montage of HFF-iPSC dfferentiation into germ cell lineages

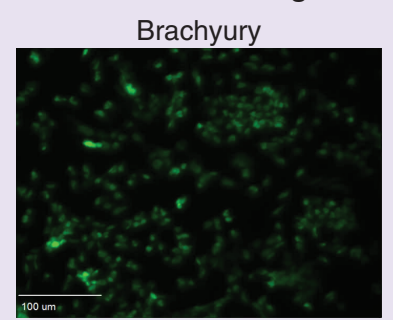

FOXA2

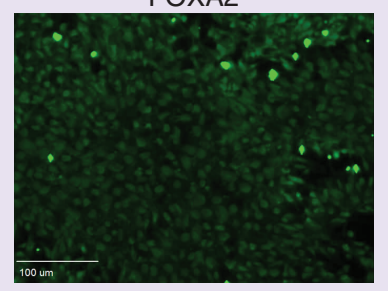

Cardiac troponin

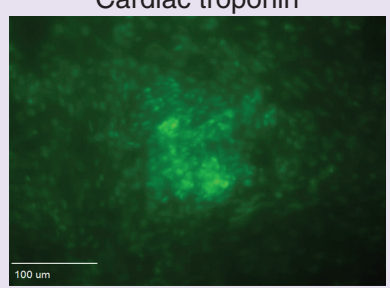

PAX6

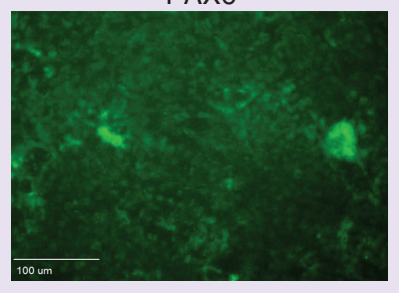

Sox17

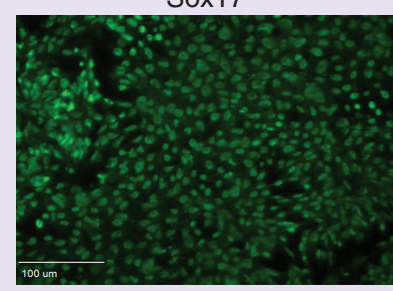

Nestin

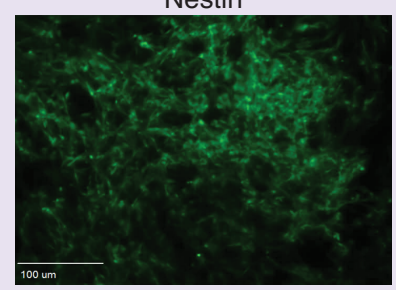

Figure 2. Montage documenting the differentiation of cultured induced pluripotent stem cell reprogrammed from cultured induced pluripotent stem cell reprogramming of cultured human foreskin fibroblast free of Myc and Lin28. iPSC were differentiated into all three germ cell lineages. Representative images show differentiation of cultured induced pluripotent stem cells into mesoderm-derived cells based on expression of brachyury (a biomarker of mesoderm) and cardiomyocytes based on cardiac troponin expression. Representative images show differentiation of cultured HFF-iPSC into definitive endoderm cells based on expression of Sox17 and FoxA2. Representative images show differentiation of cultured HFF-induced pluripotent stem cells into ectoderm-derived neuroprogenitor cells based on expression of Pax6 and Nestin. Each figure is representative of four separate experiments. Scale bar represents 100 microns.

HFF-iPSC: Induced pluripotent stem cell reprogramming of cultured human foreskin fibroblast.

\section{Myc- \& Lin28-free iPSC reprogramming of CBDMNC \& differentiation into germ cell lineages}

We further confirmed that the virus-free and Myc and Lin28-free iPSC reprogramming was robust enough to reprogram CBDMNC without purification of CD34+ cells. CBDMNC were exposed to HSC expansion media. CBDMNC were subjected to the timeline depicted in Figure 1. Figure 3B represents a montage of images of Myc/Lin28-free iPSC colonies depicting iPSC morphology and pluripotent biomarkers. Myc/Lin28-free iPSC colonies derived from CBDMNC exhibited the typical flat shape and refractile border as shown under phase microscopy (Figure 3B). iPSC colonies also stain positive for alkaline phosphatase (Figure 3B). Colonies also expressed pluripotent biomarkers that include SSEA4, Nanog, Oct4 and TRA160 (Figure 3B), which confirm that the reprogramming process resulted in pluripotent stem cells.

Myc and Lin28-free iPSC derived from CBDMNC were further differentiated into germ cell lineages (Figure 3C). iPSC were differentiated into cardiac cells expressing cardiac troponin. Cardiac differentiated cells also exhibited spontaneous beating cells (data not shown). Cultured iPSC were also serially differentiated into DE, anterior foregut endoderm and finally into lung progenitor cells. Cells were stained for expression of cytokeratin $5 / 8$, which suggested differentiation into lung progenitor cells. CD34+ iPSC were also differentiated into neuroprogenitor cells which is depicted in a phase image and an immunofluorescent image that was probed for Nestin. Taken together, these data demonstrate that the virus-free and oncogene-free iPSC reprogramming method produced pluripotent cells from parental CBDMNC and HFF.

\section{Quantification of Myc- \& Lin28-free iPSC reprogramming efficiency in CBDMNC}

HSC expansion was necessary to reprogram CBDMNCs into iPSCs. The number of colonies were measured in c-Myc, l-Myc/Lin 28 and Myc/Lin28-free groups pretreated in the presence and absence of HSC expansion media. There was iPSC colony formation in all three groups pretreated with HSC expansion media. There were statistically more observed colonies in cells reprogrammed with Myc and Lin28 than in Myc/Lin28-free cells as anticipated (Figure 4). However, there were no observed colonies in groups that were not pretreated with HSC expansion 
(A)

Montage of purified and expanded cord blood CD34+ converted into virus-free and oncogene-free iPSC

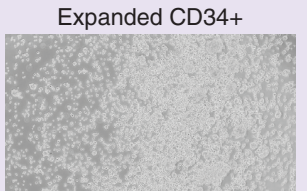

Nanog
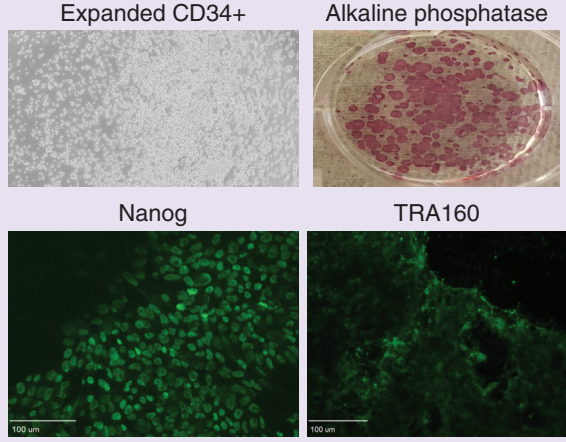

TRA160
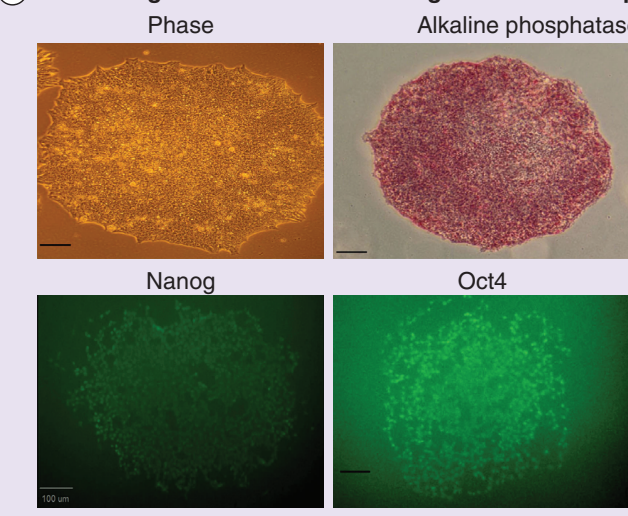

Montage image of virus-free and oncogene-free CD34-iPSC differentiated into germ cell lineages

(C)

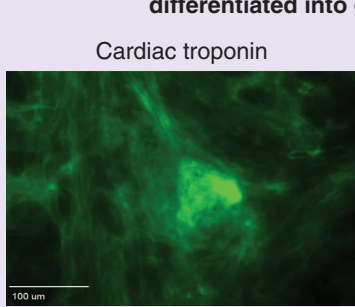

NPC-phase image

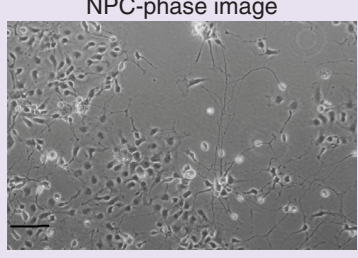

DE-derived $\mathrm{K} 5 / \mathrm{K} 8$

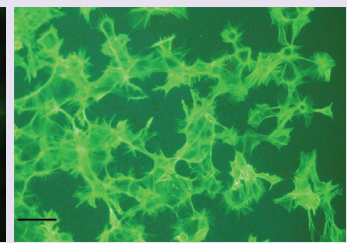

NPC-nestin

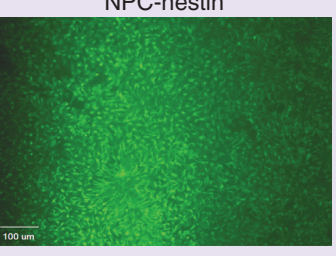

SSEA4

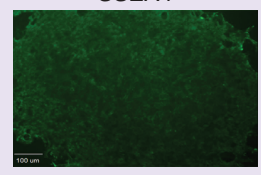

Oct4
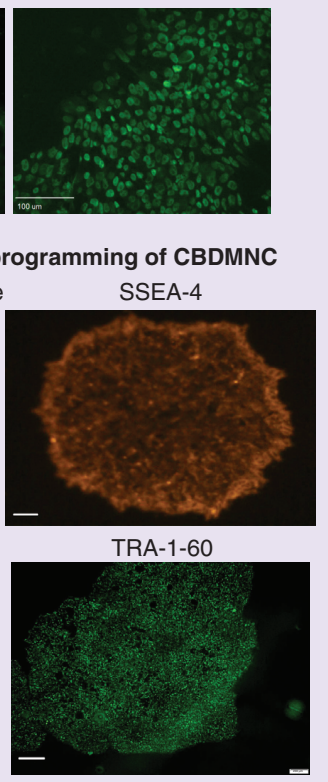

Figure 3. (A) Montage of purified and expanded cord blood CD34+ converted into virus-free and oncogene-free iPSC: CD34+ cells were positively selected in cord blood from normal donors by robotic magnetic cell sorting; further exposed to HSC expansion media for 1 week and then subjected to Myc/Lin28-free iPSC reprogramming as previously described. The montage demonstrates CD34+ cell expansion; image of a representative culture of iPSC colonies stained for alkaline phosphatase staining; and images of representative expression of SSEA4, Nanog, Oct4 and TRA160. Each figure is representative of three separate experiments. Scale bar represents 100 microns.

(B) Montage of cultured CBDMNC reprogrammed into iPSC with episomal vectors free of Myc and Lin28 and iPSC reprogramming molecules and HSC expansion media. Images were captured at day 14 of the iPSC reprogramming process. Typical iPSC colony depicted by phase contrast microscopy. Representative iPSC colony stained for alkaline phosphatase. Representative iPSC colony exhibited pluripotency by immunofluorescent live stain for SSEA4, Nanog, Oct4 and TRA160. Each figure is representative of four separate experiments. Scale bar represents 100 microns.

(C) Montage image of virus-free and oncogene-free CD34-iPSC differentiated into germ cell lineages. Virus-free and oncogene-free iPSC were differentiated into cardiac cells expressing cardiac troponin, lung progenitor cells expressing cytokeratin 5/8; and neuroprogenitor cells (phase image) and cells expressing nestin by immunofluorescence. Each figure is representative of three separate experiments. Scale bar represents 100 microns.

CBDMNC: Cord blood-derived mononuclear cell; HSC: Hematopoietic stem cell; iPSC: Induced pluripotent stem cell. 


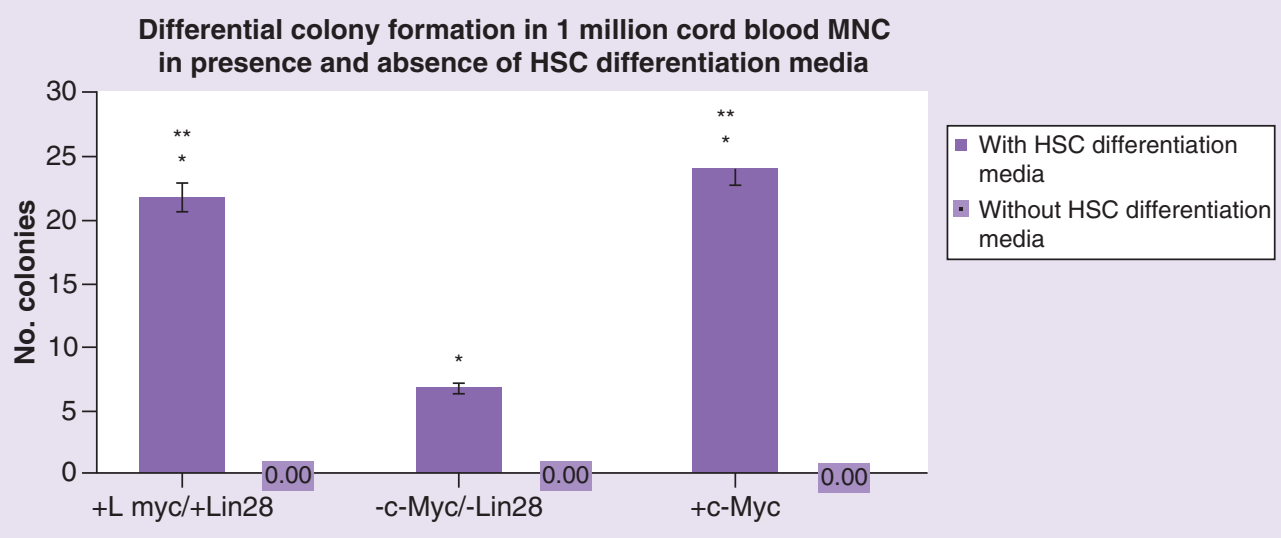

Figure 4. Figure illustrates the numbers of colonies generated between the different vector constructs among cultured cord blood-derived mononuclear cell reprogrammed in the presence and absence of hematopoietic stem cell differentiation media pretreatment. Data are reported as the mean ( \pm SE) number of colonies observed for cultured CBDMNC reprogrammed with L-Myc/Lin28, c-Myc and in the absence of both oncogenes. Each test condition used $1,000,000$ input cells. Each group represents a sample size of 4 . Data labeled with '*' show a statistical significant difference $(p<0.05)$ between cultured cells treated in the presence and absence of HSC differentiated media. Data labeled with '**' depicts a significant difference in colony formation between Myc and Lin28 and those cells treated without Myc and Lin28.

CBDMNC: Cord blood-derived mononuclear cell; HSC: Hematopoietic stem cell; MNC: Mononuclear cell.

media regardless of the presence and absence of Myc/Lin28. Taken together, these data indicate that CBDMNC conversion into iPSC required pretreatment with HSC expansion media.

HSC conversion from CBDMNC was further quantitated by flow cytometry. Cultured CBDMNC were exposed to 7 days of HSC expansion media and the amount of HSC were quantified by an antibody against human CD34+ cell surface expression. A dot blot is illustrated in Figure 5A which identified two separate populations of CD34+ cells and CD34- cells. Figure 5B depicts a histogram which demonstrated that $13 \%$ of the total cell population represented CD34+ cells. In contrast, CD34+ cells represented only $1 \%$ of unstimulated cultured CBDMNC (Figure 5C). This data supports the notion that CBDMNC expansion of the CD34+ cell pool is a prerequisite for successful iPSC reprogramming. Furthermore, this data indicates only a small a fraction of the total cellular population was necessary to achieve iPSC conversion.

Colony formation from the reprogramming of $\mathrm{CBDMNC}$ was a function of the input cell number. The number of CBDMNC-iPSC colonies created were compared in the presence and absence of Myc and Lin28 (Figure 6) at input cell numbers between 100,000 and 1,000,000. There were no iPSC colonies formed at 100,000 input cells. A minimum of 300,000 input cells was necessary to produce iPSC colonies both in the presence and absence of Myc and Lin28. A statistically greater number of iPSC colonies were observed at input cells numbers of 500,000 and $1,000,000$ compared with an input of 300,000 cells both in the presence or absence of Myc and Lin28. Consistent with the previous report [15], there was an anticipated statistically significant and greater number of colonies in Myc- and Lin28- treated cells than cells treated in the absence of these oncogenes (Figure 6).

However, the maximum reprogramming efficiency was observed at 300,000 input cells when expressed as the percentage of colonies per number of CBDMNC input number (Figure 7), regardless of the presence or absence of Myc and Lin28. As anticipated, the reprogramming efficiency was statistically higher in CBDMNC that were reprogrammed with Myc and Lin28 than in the absence of these oncogenes. When normalized to the estimated number of CD34+ cells determined by flow cytometry (13\%), the calculated reprogramming efficiencies for $\mathrm{c}-\mathrm{Myc}+, 1-\mathrm{Myc}+/ \mathrm{Lin} 28+$ and Myc/Lin28-free treated cells were $0.28,0.022$ and $0.008 \%$, respectively, at an input of 300,000 cells (Table 2). Taken together, the reprogramming efficiency is higher when normalized to the estimated CD34+ cell number than when compared with the raw CBDMNC count.

Despite the differential colony counts and reprogramming efficiency in cultured CBDMNC treated in the presence and absence of Myc and Lin28, there was no significant difference in the percentage of colonies that were fully reprogrammed. Based on the expression of SSEA4, 100\% of all colonies expressed SSEA4 with a standard 


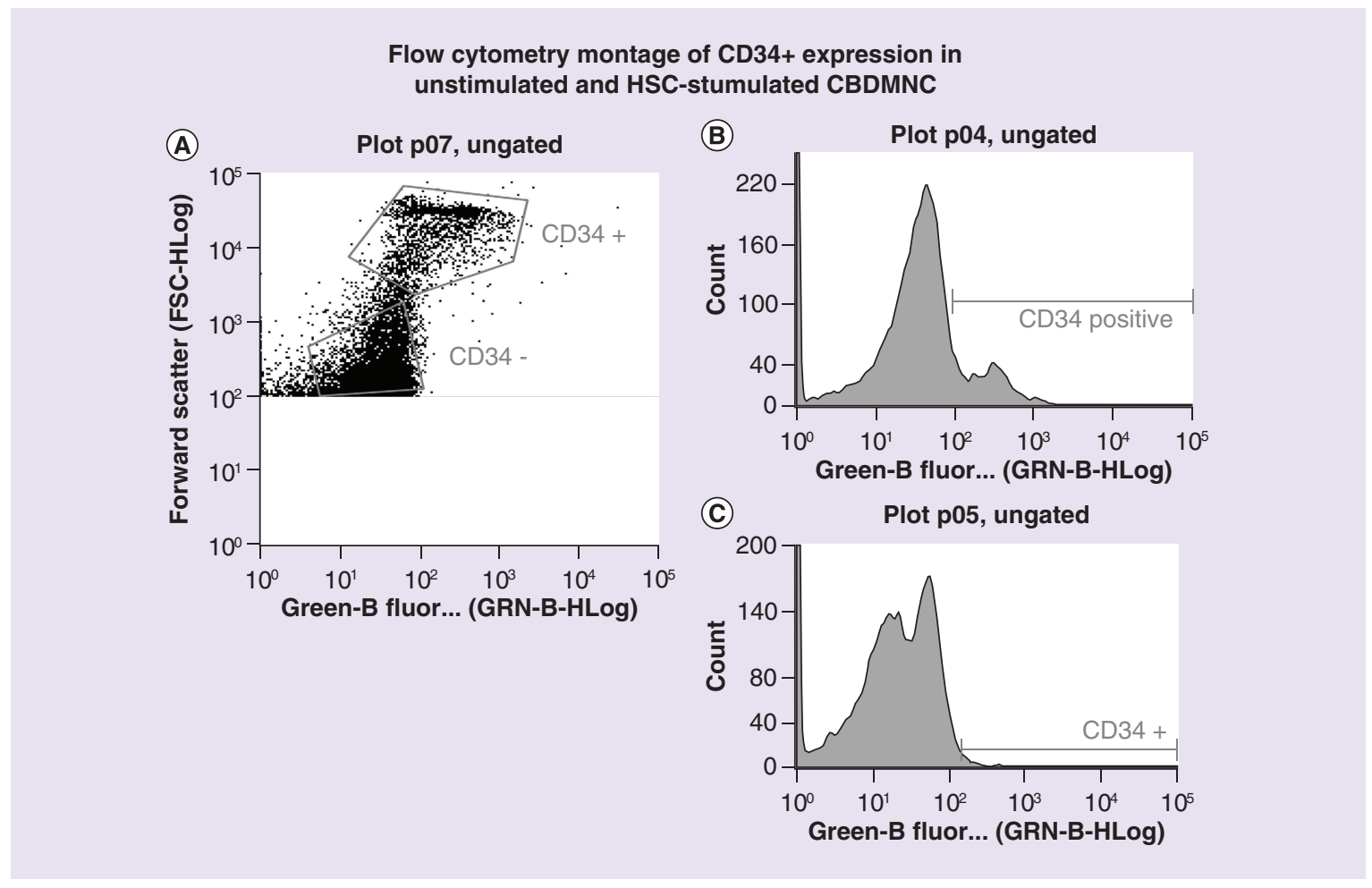

Figure 5. Data represents hematopoietic stem cell conversion from cord blood-derived mononuclear cell quantified by flow cytometry in the presence of hematopoietic stem cell expansion media. Cultured CBDMNC were exposed to 7 days of HSC expansion media and the amount of HSC expansion was quantified by an antibody specific for human CD34+ cell expression. A dot plot is illustrated in (A) depicts CD34+ and CD34- cell populations after 7 days of exposure to HSC expansion media. (B) depicts a histogram demonstrating a 13\% conversion of CBDMNC into CD34+ cells after 7 days of exposure to HSC expansion media. In contrast, CD34+ cells represented only $1 \%$ of the population of unstimulated cultured CBDMNC at baseline (C).

CBDMNC: Cord blood-derived mononuclear cell; HSC: Hematopoietic stem cell.

\begin{tabular}{|c|c|c|c|c|}
\hline & & c-Myc+ (\%) & I-Myc/Lin28+ (\%) & Myc/Lin28-free (\%) \\
\hline \multirow[t]{2}{*}{300,000 input CBDMNC } & Raw efficiency & 0.0037 & 0.0029 & 0.0010 \\
\hline & Normalized efficiency & 0.0282 & 0.0222 & 0.0077 \\
\hline \multirow[t]{2}{*}{500,000 input CBDMNC } & Raw efficiency & 0.0025 & 0.0019 & 0.0009 \\
\hline & Normalized efficiency & 0.0190 & 0.0149 & 0.0067 \\
\hline \multirow[t]{2}{*}{$1,000,000$ input CBDMNC } & Raw efficiency & 0.0024 & 0.0022 & 0.0007 \\
\hline & Normalized efficiency & 0.0185 & 0.0167 & 0.0051 \\
\hline
\end{tabular}

The normalized reprogramming efficiency is calculated by estimating the total CD34+ cells based on the flow cytometry data from Figure 5 . Data are reported as the mean observed for cultured CBDMNC reprogrammed with I-MyC/Lin28, C-Myc and in the absence of both oncogenes with 300,000, 500,000 and 1,000,000 input CBDMNC.

CBDMNC: Cord blood-derived mononuclear cell.

error $=0$ (Figure 8). These effects were consistent at inputs of 300,000, 500,000 and 1,000,000 cells. Taken together, there was no advantage in the reprogramming efficiency with cultured CBDMNC tested at cell input numbers that exceeded 300,000 cells.

\section{iPSC reprogramming of PBMNC of CF \& A1ATD patients}

We further confirmed that the virus-free and oncogene-free iPSC reprogramming method was robust enough to create patient and disease-specific iPSC models from PBMNC from patients with genetic lung disease. PBMNC 


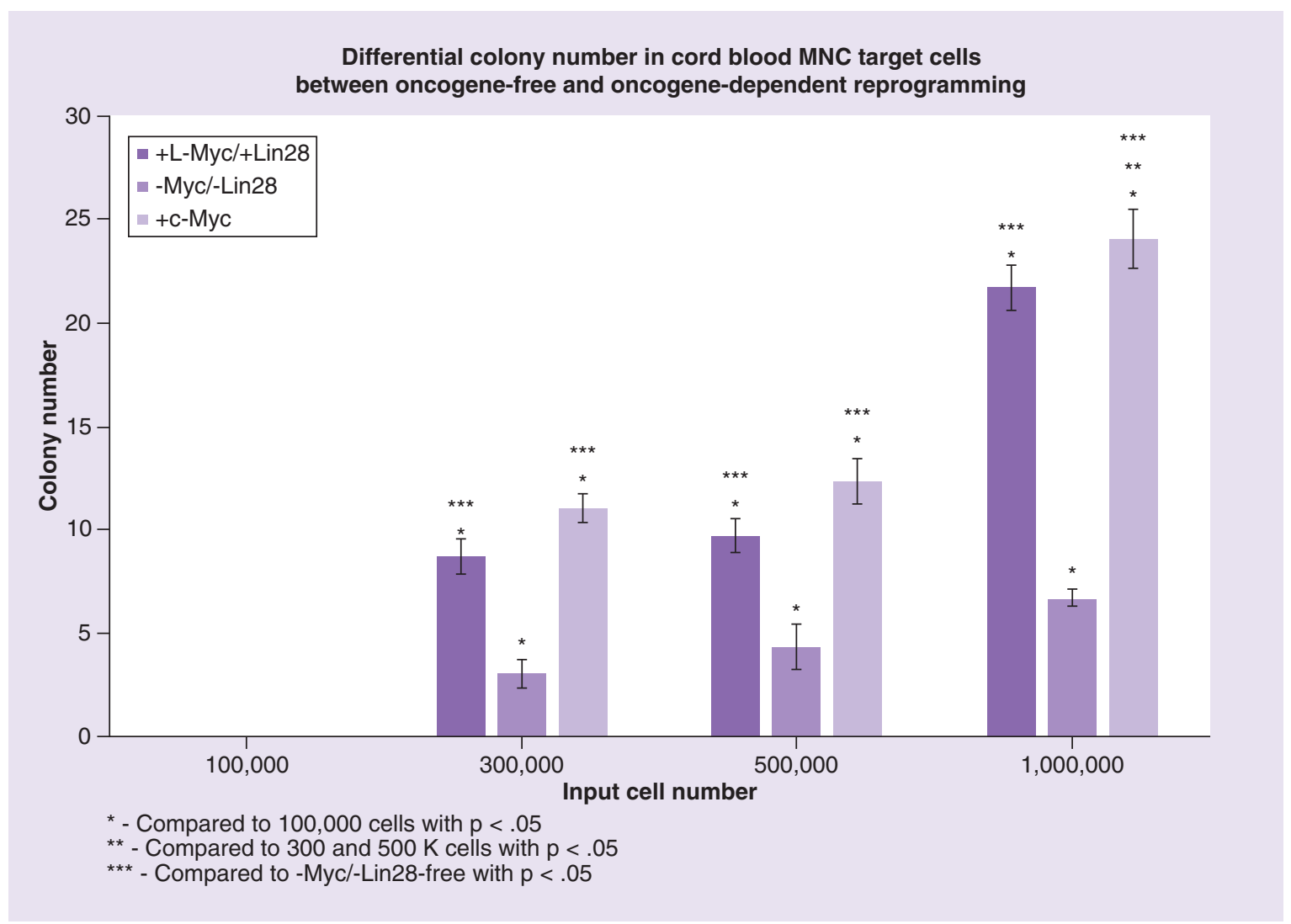

Figure 6. The number of induced pluripotent stem cell colonies created in the presence of hematopoietic stem cell differentiation media increase as a function of the input number of cord blood-derived mononuclear cell. Figure illustrates the numbers of colonies generated between the different vector constructs among cultured CBDMNC reprogrammed in the presence of HSC differentiation media and reprogramming molecules. Data are reported as the mean $( \pm$ SE) number of colonies observed for cultured CBDMNC reprogrammed with L-Myc/Lin28, c-Myc and in the absence of both oncogenes from 100,000,300,000,500,000 and 1,000,000 input cells. Each group represents a sample size of 4. Data labeled with '*' show a statistical significant difference $(p<0.05)$ in colony number between cultured cells treated with Myc and Lin28 and those cells treated without Myc and Lin28 at the same corresponding input cell number.

CBDMNC: Cord blood-derived mononuclear cell; HSC: Hematopoietic stem cell.

were reprogrammed into iPSC according to the timeline described in Figure 1. Upon exposure to HSC expansion media, there was increased cells that had a HSC-like morphology (data not shown). Figure 9 represents a montage of a typical iPSC colony and corresponding pluripotent biomarkers from a 57-year-old white female with A1ATD expressing a $\mathrm{ZZ}$ phenotype. The montage depicts a representative phase image of an iPSC colony with flat surface and refractile borders; an alkaline phosphatase-stained colony; and representative colonies stained for Nanog, Oct-4, SSEA-4 and TRA-1-60.

A virus-free and oncogene-free iPSC was created with PBMNC from a 7-year-old male CF patient with a homozygous mutation at the $\delta-508$ position. The following montage of images confirm that the iPSC are pluripotent (Figure 10), which demonstrate: an image of the entire colonies; representative phase image of a single alkaline phosphatase stained colony; colonies stained for Nanog expression; colonies stained for Oct-4 expression; colonies stained for SSEA4 expression; and colonies stained for TRA-1-60 expression.

\section{Discussion}

This study validates a virus-free and oncogene-free method of reprogramming cord blood and peripheral blood derived MNC into iPSC, which has the potential for a safer autologous pluripotent cell therapy and potentially a more native and predictive patient-specific iPSC for drug development. This study extends previous work on adherent cells and optimized episomal-based Myc and Lin28-free iPSC reprogramming method to convert MNC 


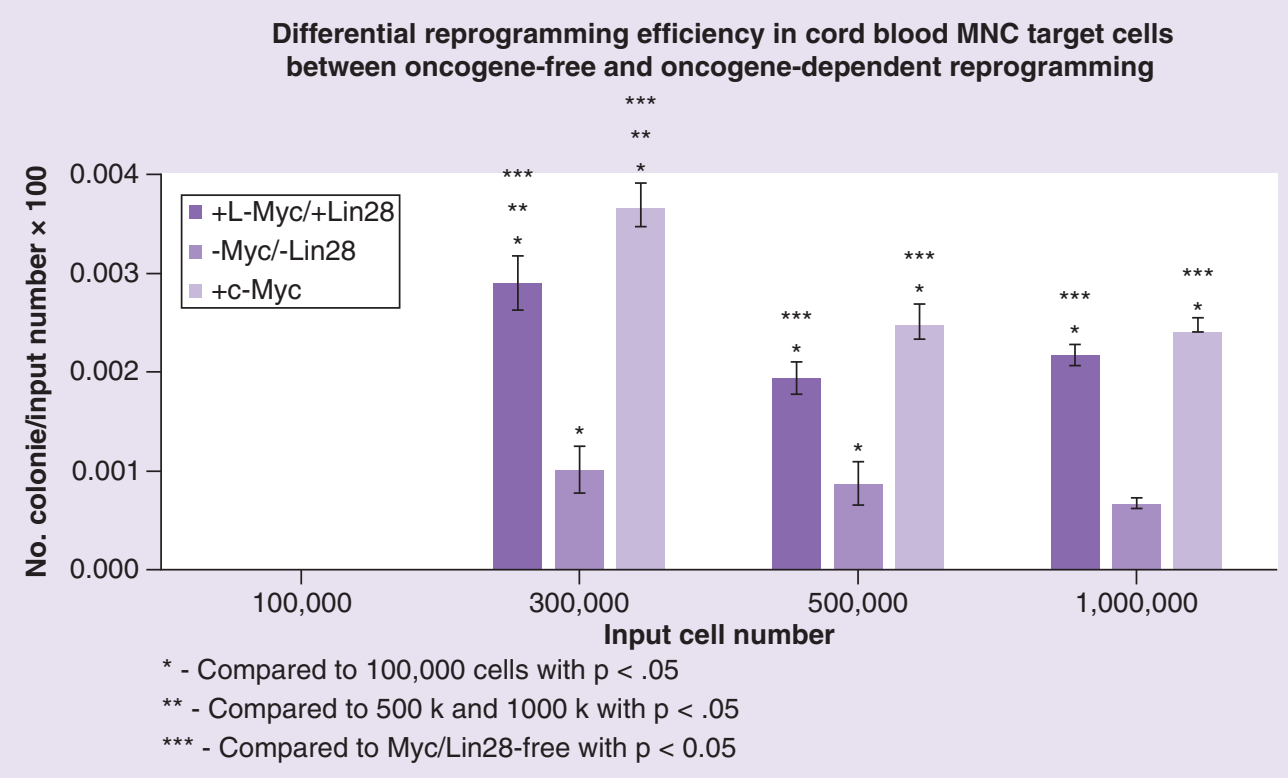

Figure 7. Figure illustrates the reprogramming efficiency generated between the different vector constructs among cultured cord blood-derived mononuclear cell reprogrammed in the presence of hematopoietic stem cell differentiation media and reprogramming molecules. Data are reported as the mean $( \pm S E)$ reprogramming efficiency observed for cultured CBDMNC reprogrammed with L-Myc/Lin28, c-Myc and in the absence of both oncogenes with $100,000,300,000,500,000$ and 1,000,000 input cells. Each group represents a sample size of 4 . Data labeled with '*' show a statistical significant difference $(p<0.05)$ in reprogramming efficiency between cultured cells treated with Myc and Lin28 and those cells treated without Myc and Lin28 at each corresponding input cell number. CBDMNC: Cord blood-derived mononuclear cell; MNC: Mononuclear cell.

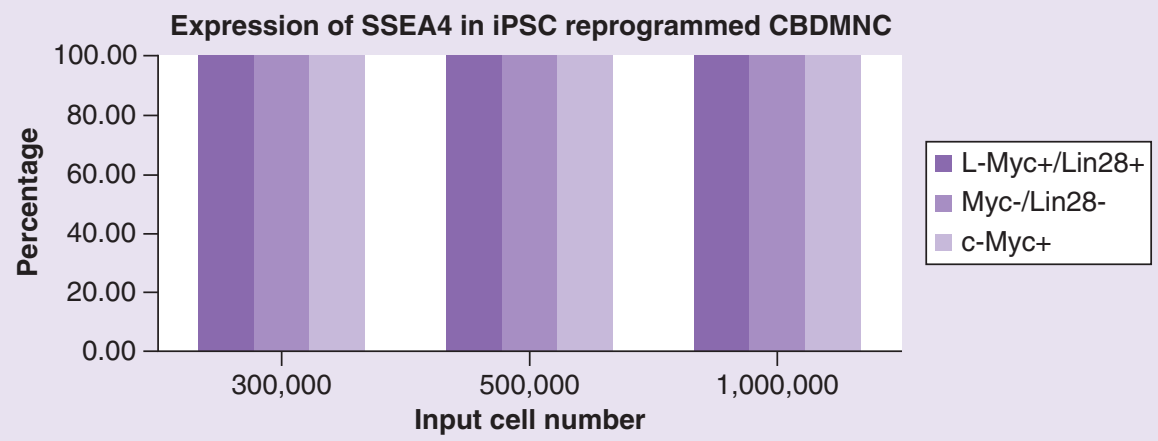

Figure 8. Induced pluripotent stem cellcolonies from cord blood-derived mononuclear cell conversion with reprogramming molecules and hematopoietic stem cell expansion media are all fully reprogrammed regardless of the presence or absence of Myc/Lin28. Figure depicts the percentage of colonies that express SSEA4 among cultured CBDMNC exposed to I-Myc/Lin28, c-Myc and the absence of both oncogene groups. Data are reported as the mean (the standard error $=0$ ). All colonies stained positive for SSEA4. Each group represents a sample size of four replicates. Full pluripotency was achieved at 300,000, 500,000 and 1,000,000 input cells.

CBDMNC: Cord blood-derived mononuclear cell; iPSC: Induced pluripotent stem cell.

from cord blood and peripheral blood. This report demonstrates that MNC convert into iPSC only after exposure to HSC expansion media that increases the pool of CD34+ cells and subsequent treatment with reprogramming small molecules in which the latter was previously reported [15]. This approach is efficient in reprogramming MNC from cord blood and from peripheral blood in patients who have genetic-based chronic lung disease. A previous report documented an iPSC reprogramming method in cultured adherent cells, which uses a combinatorial 


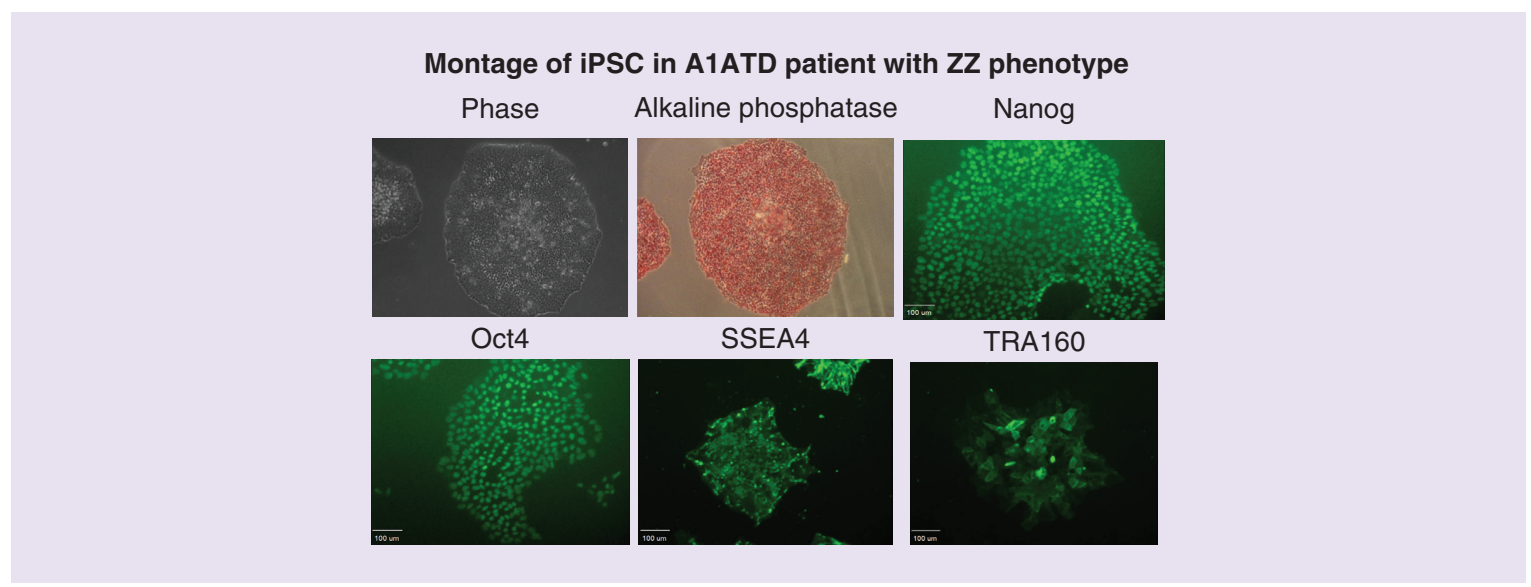

Figure 9. Montage of cultured induced pluripotent stem cell reprogrammed with peripheral blood mononuclear cell from a 57-year-old Caucasian female with $\alpha-1$ antitrypsin deficiency with a PiZZ phenotype. Cells were reprogrammed into iPSC with episomal vectors free of Myc and Lin28 and HSC expansion media. Images were captured at day 14 of the iPSC reprogramming process. Typical iPSC colony depicted by phase contrast microscopy. Representative iPSC colony stained positive for alkaline phosphatase. Representative iPSC colonies exhibited pluripotency by immunofluorescent live stain for SSEA4, Nanog, Oct4 and TRA160. Each figure is representative of four separate experiments.

HSC: Hematopoietic stem cell; iPSC: Induced pluripotent stem cell.

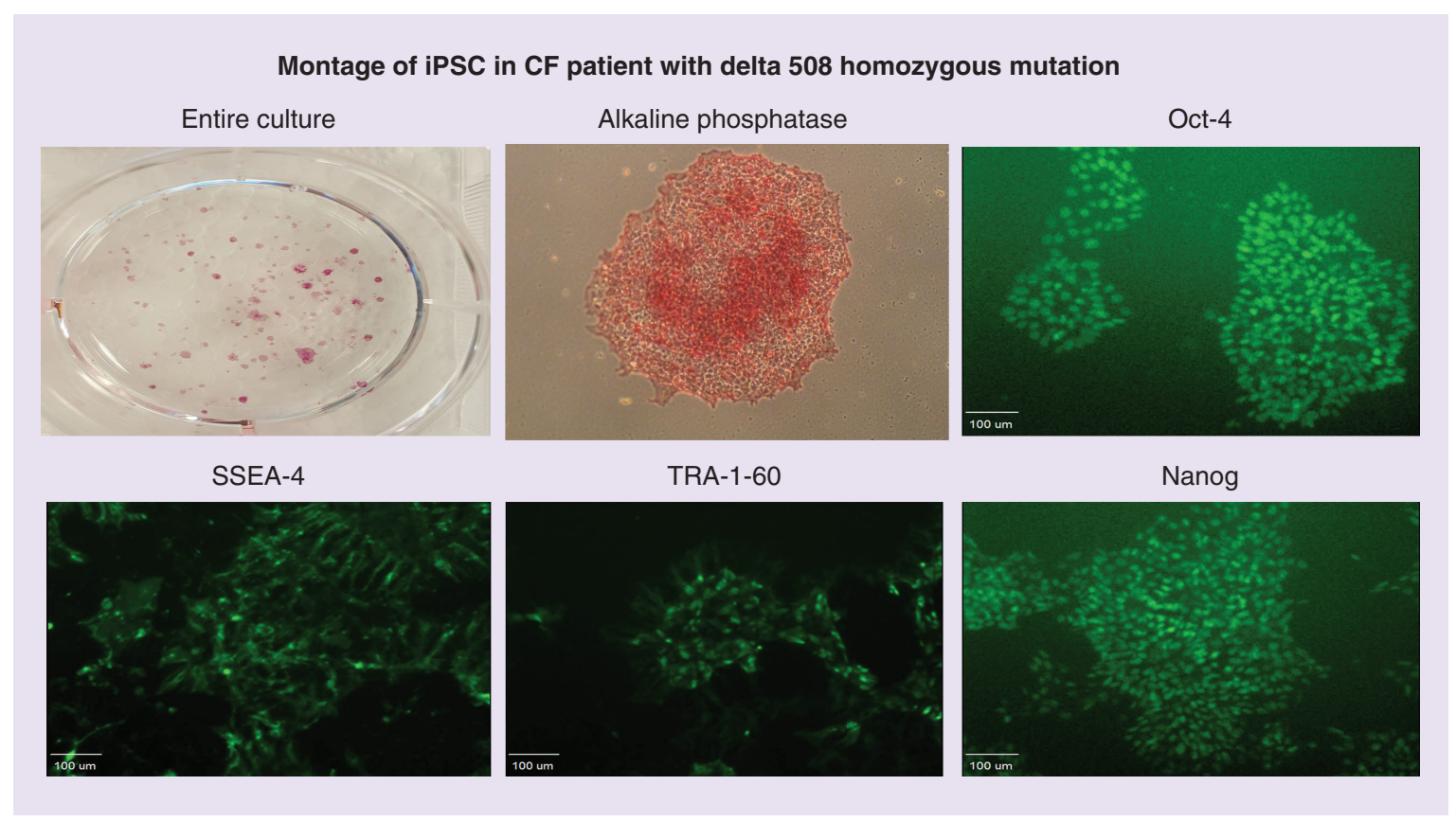

Figure 10. Montage of cultured induced pluripotent stem cell reprogrammed from peripheral blood mononuclear cell among a 7-year-old Caucasian male with cystic fibrosis with the $\delta$-508 mutation. Cells were reprogrammed into iPSC with episomal vectors free of Myc and Lin28 and HSC expansion media. Images were captured at day 14 of the iPSC reprogramming process. Representative image of an entire culture stained with alkaline phosphatase.

Representative iPSC colony stained for alkaline phosphatase. Representative iPSC colonies exhibited pluripotency by immunofluorescent live stain for SSEA4, Nanog, Oct4 and TRA160. Each figure is representative of four separate experiments. Scale bar represents 100 microns.

CF: Cystic fibrosis; HSC: Hematopoietic stem cell; iPSC: Induced pluripotent stem cell. 
approach of reprogramming small molecule enhancers and a mixture of episomal vectors that are free of Myc and Lin28 [15]. However, iPSC reprogramming of suspension cells is known to be less efficient than adherent cells [18-21]. iPSC reprogramming requires phenotypic transitioning from a mesenchymal to a transient epitheliallike phenotype [22,23]. Thus, a robust nonviral, nonintegrating and Myc- and Lin28-free iPSC reprogramming method is needed to expand the stem cell plasticity and scalability of cord blood for cell therapy as well as to develop patient and disease-specific disease cell models. Using a minimally invasive approach like a venipuncture to genetic reprogram PBMNC from infants and children would be preferable to dermal fibroblasts which requires a punch skin biopsy.

The objective of the study was to optimize the Myc- and Lin28-free iPSC reprogramming method for circulating MNC by using the combinatorial approach of episomal vectors and reprogramming molecules. Pretreatment of MNC with HSC expansion media was necessary for iPSC reprogramming. We did not observe any iPSC colony formation in the absence of HSC expansion. Thus, pretreatment of cultured MNC with HSC expansion media was used as a standard operating procedure in all experiments for reprogramming CBDMNC and PBMNC.

The minimum number of input CBDMNC to produce iPSC colonies was 300,000. Unlike a prior report, which used 100,000 input cultured fibroblasts, reprogramming CBDMNC required three-times more input cells than to reprogram cultured HFF [15]. A dose-dependent increase in colony formation was observed with increasing number of input cells whether in the presence or absence of Myc and Lin28. As expected, there were more colonies formed with Myc and Lin28 than in the absence of these oncogenes at each level of input cells.

However, when iPSC reprogramming was quantified as a function of reprogramming efficiency, the maximal efficiency was observed at 300,000 input cells. Furthermore, the calculated iPSC reprogramming efficiency was higher when corrected for the number of CD34+ cells during HSC expansion. The number of CD34+ cells were calculated based on the fraction of CD34+ cells measured by flow cytometry. The HSC expansion media increased the fraction of CD34+ cells from 1\% under unstimulated conditions to $13 \%$ after 7 days of exposure to HSC expansion media. When corrected for the CD34+ cell count, the calculated iPSC reprogramming efficiency was seven-times greater than when the reprogramming efficiency was based on the MNC count. The corrected iPSC reprogramming efficiency is consistent with the reprogramming efficiency previously reported in adherent cells [15]. The reason for why the reprogramming efficiency is greatest at 300,000 input CBDMNC is not clear. One possible explanation might be due to the optimal mix of cell number, DNA constructions and appropriate small molecule concentration.

While the transfection of 1-Myc and c-Myc mediated a greater number of colonies than oncogene-free reprogramming, oncogene-free iPSC colonies were fully reprogrammed irrespective between 300,000 to $1,000,000$ input CBDMNC. Thus, the reprogramming method showed that colonies were fully reprogrammed irrespective of whether transcriptional oncogenes were used or not. Since cultured cells were treated with an excess of exogenous DNA, more colonies were formed at a higher input cell number but without an associated increase in reprogramming efficiency. Thus, the combination of reprogramming molecules and HSC expansion media served as a catalyst for successfully reprogramming of circulating MNC without the need to use Lin28- and Myc-dependent transcriptional factors.

The virus-free and oncogene-free iPSC reprogramming method demonstrated pluripotency in iPSCs derived from cultured HFF and from cord blood-specific CD34+ cells. Each iPSC cell differentiated into all three germ cell lineages. However, we cannot rule out that there are quantitative and qualitative differences in cell phenotype between iPSC derived from different parental cells. Furthermore, it is not known whether there could be differences in genetic instability between oncogene-free and oncogene-dependent iPSC during large scale cell expansion for cell therapy, which has been previously described in oncogene-dependent iPSC [24]. Additionally, it is not known whether there are phenotypic differences in iPSC-differentiated cells between cells reprogrammed in the presence and absence of oncogenes. Such differences could have important effects on the efficacy of cell therapy and on patient-specific cell lines in predicting clinical pharmacological responses. Yet, this virus-free and oncogene-free approach now allows, for the first time, the opportunity to directly test these hypotheses.

The combinatorial approach of oncogene-free iPSC reprogramming, which uses small molecules and HSC expansion media, was robust enough to create patient and disease-specific iPSC lines from peripheral blood taken from patients with CF and A1ATD. Oncogene-free and virus-free reprogramming methodology provides patient and disease-specific iPSC for drug discovery and personalized medicine applications with lower risk of oncogenic and viral perturbations that could skew the predictability of cell models of disease. Residual transgenes from reprogramming has been reported to alter the phenotype of iPSC-differentiated cells [25]. Additionally, iPSC 
reprogramming with c-Myc mediated repression of cardiac differentiation compared with c-Myc-free iPSC cells [26]. A virus-free and oncogene-free in vitro patient-specific cell assay could improve the predictability of drug screening to identify lead drugs. Also, a virus-free and oncogene-free in vitro patient-specific CF cell model could help stratify patients in clinical trials and decrease the inherent high attrition in drug development [27,28]. Also, PBMNC-derived A1ATD and CF-iPSC could lead to autologous cell therapy when coupled with gene editing. To the best of our knowledge, this report represents the first virus-free and oncogene-free iPSC models from CF and A1ATD patients.

We chose episomal vectors and avoided Myc and Lin 28 over other nonintegrating methods such as the Sendai virus because of the lower therapeutic risk by avoiding the use of viral gene delivery. Sendai virus-based reprogramming requires far more cell divisions to dilute viral proteins from detection. Also, cell lines must be screened for viral protein remnants before being introduced as cell therapy, which increases the time and cost of cell therapy manufacturing. Whatever perceived advantages Sendai virus may have on the front end in terms of reprogramming efficiency will be lost on the back end in cell therapy production in terms of time and cost. In contrast, episomal vectors are eliminated sooner and require no screening for viral elements and the cost is far less expensive as a manufacturing approach.

The innovation introduced in this report is underscored by solving the low efficiency and inconsistency of episomal iPSC reprogramming in cord blood and peripheral blood. Furthermore, the reprogramming method was optimized free of c-Myc and Lin28. The safety concern of iPSC-based therapy centers on the neoplastic risk that could be introduced into a patient if residual undifferentiated iPSC remains in the final product. Since manufacturing processes are rarely $100 \%$ efficient, even a $1 \%$ contamination of undifferentiated iPSC could result in a significant number of pluripotent stem cells at systemic doses on the order of 1-2 million cells per $\mathrm{kg}$ of body weight. Thus, the need for c-Myc and Lin28-free iPSC technology is critical.

Last, the technology creates a regulatory pathway for large scale manufacturing of iPSC-derivative cell therapies for a variety of chronic diseases in which the manufacturing process should reduce infectious and oncogenic risk. The reprogramming method paves a pathway for autologous and allogeneic cell therapy that satisfies regulatory requirements. The reprogramming method for converting circulatory MNC into iPSC is cost effective, efficient and provides an extremely high yield of pluripotent conversion and purity.

\section{Conclusion}

The combination of episomal vectors and mixture of reprogramming molecules produce an efficient scheme for creating iPSC from cord blood and peripheral blood derived MNC without the need to introduce Myc and Lin28 once they are pretreated with HSC expansion molecules.

\section{Translational perspective}

This report represents a milestone by providing a potentially safer pluripotent stem cell therapy than prior iPSC reprogramming methods that have required Myc and Lin28. Further, the ability to reprogram cord blood and peripheral blood using a virus-free and oncogene-free reprogramming strategy now offers the opportunity to transition this technology into a manufacturing operation to produce cell therapy for a variety of chronic diseases. This technology could solve the shortcomings of stored cord blood in both private and public banks used for regenerative medicine applications. Cord blood conversion into iPSC could increase the diversity and dosage necessary for autologous and allogeneic cell therapy. Additionally, the iPSC reprogramming method could combine with gene editing methods to create autologous cell and gene therapy from peripheral blood. Additionally, this new oncogenic-free reprogramming approach could potentially deliver targeted cell therapy through in vivo as well as ex vivo differentiation process. 


\section{Summary points}

- Mixture of virus-free and oncogene-free induced pluripotent stem cell (iPSC) reprogramming molecules and hematopoietic stem cell (HSC) expansion media efficiently reprograms purified cord blood-derived CD34+ cells.

- Mixture of virus-free and oncogene-free iPSC reprogramming molecules and HSC expansion media also efficiently reprograms cord blood-derived mononuclear cells and peripheral blood mononuclear cells (in patients with cystic fibrosis and $\alpha-1$ anti-trypsin deficiency) without need for CD34+ purification.

- Cord blood-derived mononuclear cell reprogramming was dependent on the input cell number and was most efficient at 300,000 cells upon exposure to HSC expansion media.

- $100 \%$ of reprogrammed colonies in Myc and Lin28-free cells expressed SSEA4 in the presence of reprogramming molecules and prior HSC expansion.

- Virus-free and oncogene-free iPSC reprogramming exhibit pluripotency based on expression of pluripotent biomarkers and differentiation in all germ cell lineages in human foreskin fibroblasts and cord blood-derived CD34+ cells.

- The iPSC reprogramming method is virus-free, integration-free, matrigel-free, oncogene-free (Myc- and Lin28-free) and feeder-free, which meets regulatory requirements for developing derivative autologous and allogeneic iPSC therapy with potentially lower infectious and neoplastic risk from peripheral and cord blood.

\section{Open access}

This work is licensed under the Attribution-NonCommercial-NoDerivatives 4.0 Unported License. To view a copy of this license, visit http://creativecommons.org/licenses/by-nc-nd/4.0/

\section{Ethical conduct}

The authors state that they have obtained appropriate institutional review board approval or have followed the principles outlined in the Declaration of Helsinki for all human or animal experimental investigations.

\section{References}

1. Shearer WT, Lubin BH, Cairo MS, Notarangelo LD. Cord blood banking for potential future transplantation. Pediatrics 140(5), 2017-2695 (2017).

2. Shlush LI, Zandi S, Mitchell A et al. Identification of pre-leukaemic haematopoietic stem cells in acute leukaemia. Nature 506(7488), 328-333 (2014).

3. Delaney C, Bollard CM, Shpall EJ. Cord blood graft engineering. Biol. Blood Marrow Transplant. 19(1 Suppl), S74-S78 (2013).

4. Seita J, Weissman IL. Hematopoietic stem cell: self-renewal versus differentiation. Wiley Interdiscip. Rev. Syst. Biol. Med. 2(6), 640-653 (2010).

5. Peterson SE, Loring JF. Genomic instability in pluripotent stem cells: implications for clinical applications. J. Biol. Chem. 289(8), 4578-4584 (2014).

6. Takahashi K, Tanabe K, Ohnuki M et al. Induction of pluripotent stem cells from adult human fibroblasts by defined factors. Cell 131(5), 861-872 (2007).

7. Takahashi K, Yamanaka S. Induction of pluripotent stem cells from mouse embryonic and adult fibroblast cultures by defined factors. Cell 126(4), 663-676 (2006).

8. Yu J, Vodyanik MA, Smuga-Otto K et al. Induced pluripotent stem cell lines derived from human somatic cells. Science 318(5858), 1917-1920 (2007).

9. Nakagawa M, Koyanagi M, Tanabe K et al. Generation of induced pluripotent stem cells without Myc from mouse and human fibroblasts. Nat. Biotechnol. 26(1), 101-106 (2008).

10. Nakagawa M, Takizawa N, Narita M, Ichisaka T, Yamanaka S. Promotion of direct reprogramming by transformation-deficient Myc. Proc. Natl Acad. Sci. USA 107(32), 14152-14157 (2010).

11. Ikegaki N, Minna J, Kennett RH. The human L-myc gene is expressed as two forms of protein in small cell lung carcinoma cell lines: detection by monoclonal antibodies specific to two myc homology box sequences. EMBO J. 8(6), 1793-1799 (1989).

12. Bektas-Kayhan K, Unur M, Yaylim-Eraltan I et al. Role of L-MYC polymorphism in oral squamous cell carcinoma in Turkey. Anticancer Res. 29(7), 2519-2524 (2009).

13. Yaylim-Eraltan I, Bozkurt N, Ergen A et al. L-myc gene polymorphism and risk of thyroid cancer. Exp. Oncol. 30(2), 117-120 (2008).

14. Zhao T, Zhang ZN, Rong Z, Xu Y. Immunogenicity of induced pluripotent stem cells. Nature 474(7350), 212-215 (2011).

15. Kamath A, Ternes S, Mcgowan S, English A, Mallampalli R, Moy AB. Efficient method to create integration-free, virus-free, Myc and Lin28-free human induced pluripotent stem cells from adherent cells. Future Sci. OA 3(3), FSO211 (2017). 
16. Yu J, Hu K, Smuga-Otto K et al. Human induced pluripotent stem cells free of vector and transgene sequences. Science 324(5928), 797-801 (2009).

17. Okita K, Matsumura Y, Sato Y et al. A more efficient method to generate integration-free human iPS cells. Nat. Methods 8(5), 409-412 (2011).

18. Chou BK, Mali P, Huang X et al. Efficient human iPS cell derivation by a non-integrating plasmid from blood cells with unique epigenetic and gene expression signatures. Cell Res. 21(3), 518-529 (2011).

19. Liu J, Brzeszczynska J, Samuel K et al. Efficient episomal reprogramming of blood mononuclear cells and differentiation to hepatocytes with functional drug metabolism. Exp. Cell Res. 338(2), 203-213 (2015).

20. Meng X, Neises A, Su RJ et al. Efficient reprogramming of human cord blood CD34+ cells into induced pluripotent stem cells with OCT4 and SOX2 alone. Mol. Ther. 20(2), 408-416 (2012).

21. Munoz-Lopez A, Van Roon EH, Romero-Moya D et al. Cellular ontogeny and hierarchy influence the reprogramming efficiency of human B cells into induced pluripotent stem cells. Stem Cells 34(3), 581-587 (2016).

22. Li R, Liang J, Ni S et al. A mesenchymal-to-epithelial transition initiates and is required for the nuclear reprogramming of mouse fibroblasts. Cell Stem Cell 7(1), 51-63 (2010).

23. Hu X, Zhang L, Mao SQ et al. Tet and TDG mediate DNA demethylation essential for mesenchymal-to-epithelial transition in somatic cell reprogramming. Cell Stem Cell 14(4), 512-522 (2014).

24. Baker D, Hirst AJ, Gokhale PJ et al. Detecting genetic mosaicism in cultures of human pluripotent stem cells. Stem Cell Rep. 7(5), 998-1012 (2016).

25. Martinez-Fernandez A, Nelson TJ, Reyes $S$ et al. iPS cell-derived cardiogenicity is hindered by sustained integration of reprogramming transgenes. Circ. Cardiovasc. Genet. 7(5), 667-676 (2014).

26. Martinez-Fernandez A, Nelson TJ, Ikeda Y, Terzic A. c-MYC independent nuclear reprogramming favors cardiogenic potential of induced pluripotent stem cells. J. Cardiovasc. Transl. Res. 3(1), 13-23 (2010).

27. Hay M, Thomas DW, Craighead JL, Economides C, Rosenthal J. Clinical development success rates for investigational drugs. Nat. Biotechnol. 32(1), 40-51 (2014).

28. Dimasi JA, Feldman L, Seckler A, Wilson A. Trends in risks associated with new drug development: success rates for investigational drugs. Clin. Pharmacol. Ther. 87(3), 272-277 (2010). 
